\title{
Treatment and outcome of Ganglioneuroma and Ganglioneuroblastoma intermixed
}

Boris Decarolis ${ }^{1}$ (D) Thorsten Simon ${ }^{1}$, Barbara Krug ${ }^{2}$, Ivo Leuschner ${ }^{3}$, Christian Vokuhl $^{3}$, Peter Kaatsch ${ }^{4}$, Dietrich von Schweinitz ${ }^{5}$, Thomas Klingebiel ${ }^{6}$, Ingo Mueller ${ }^{7}$, Lothar Schweigerer ${ }^{8}$, Frank Berthold ${ }^{1}$ and Barbara Hero ${ }^{1 *}$

\begin{abstract}
Background: Ganglioneuroma (GN) and ganglioneuroblastoma intermixed (GNBI) are mature variants of neuroblastic tumors (NT). It is still discussed whether incomplete resection of GN/GNBI impairs the outcome of patients.

Methods: Clinical characteristics and outcome of localized GN/GNBI were retrospectively compared to localized neuroblastoma (NB) and ganglioneuroblastoma-nodular (GNBN) registered in the German neuroblastoma trials between 2000 and 2010.

Results: Of 808 consecutive localized NT, 162 (20\%) were classified as GN and 55 (7\%) as GNBI. GN/GNBI patients presented more often with stage 1 disease ( $68 \%$ vs. $37 \%, p<0.001$ ), less frequently with adrenal tumors (31\% vs. $43 \%, p=0.001)$ and positive mIBG-uptake (34\% vs. $90 \%, p<0.001)$, and had less often elevated urine catecholamine metabolites (homovanillic acid $39 \%$ vs. $62 \%, p<0.001$, vanillylmandelic acid $27 \%$ vs. $64 \%, p<0.001$ ). Median age at diagnosis increased with grade of differentiation (NB/GNBN: 9; GNBI: 61; GN-maturing: 71; GN-mature: 125 months, $\mathrm{p}<0.001$ ). Complete tumor resection was achieved at diagnosis in $70 \%$ of $162 \mathrm{GN}$ and $67 \%$ of $55 \mathrm{GNBI}$, and after 4 to 32 months of observation in 4 GN (2\%) and 5 GNBI (9\%). Eleven patients received chemotherapy without substantial effect. Fifty-five residual tumors ( $42 \mathrm{GN}, 13 \mathrm{GNBI}$ ) are currently under observation (median: 44 months). Five patients (3 GN, 2 GNBI) showed local progression; all had tumor residuals $>2 \mathrm{~cm}$. No progression occurred after subtotal resection. Two patients died of treatment, none of tumor progression.
\end{abstract}

Conclusions: GN/GNBI account for one quarter of localized NT and differ from immature tumors in their clinical features. Chemotherapy is not effective. Subtotal resection appears to be a sufficient treatment.

Trial registration: ClinicalTrials.gov identifiers - NB97 (NCT00017225; registered June 6, 2001); NB2004 (NCT00410631; registered December 11, 2006)

Keywords: Ganglioneuroma, Ganglioneuroblastoma intermixed, Therapy, Surgery, Subtotal resection, Treatment, Residual tumor

\footnotetext{
* Correspondence: barbara.hero@uk-koeln.de

${ }^{1}$ Department of Pediatric Hematology and Oncology, Children's Hospital,

University of Cologne, Cologne, Germany

Full list of author information is available at the end of the article
} 


\section{Background}

Neuroblastic tumors (NT) are the most common extracranial solid tumors in childhood [1] and include neuroblastoma, ganglioneuroblastoma (nodular or intermixed), and ganglioneuroma. They arise from the neural crest and range from immature, undifferentiated to mature, differentiated tumors. According to the International Neuroblastoma Pathology Classification (INPC) [2], ganglioneuroblastoma intermixed (GNBI) and ganglioneuroma $(\mathrm{GN})$ represent the mature end of this range [3]. In this system, GN maturing has been defined as a "link" between GN and GNBI.

Ganglioneuroma has been first described more than 150 years ago [4]. A variety of case reports on GN have been published [4-14], ranging from patients with symptoms due to huge tumor masses [10] to speculations about malignant transformation and dedifferentiation into neuroblastoma $[7,8]$. GN is generally considered a benign tumor that is treated by surgery alone. However, ganglioneuroblastoma intermixed (GNBI) is widely seen as a malignant entity and - depending on stage - treated with multimodal therapy. Case reports on GNBI are rarer $[15,16]$.

Only four larger series of GN and / or GNBI in pediatric patients have been reported [17-20]. In a previous analysis of our group, we assessed metabolic and clinical features of GN and demonstrated that a relevant proportion of GN shows mIBG uptake and elevated catecholamine metabolites in urine [17]. Furthermore, while complete resection was widely considered the standard treatment for $\mathrm{GN}$, this analysis suggested that incomplete resection might be sufficient for the treatment of GN [17]. This was supported by De Bernardi et al who also suggested a more cautious surgical approach to localized GNBI and proposed that GN and GNBI show similar clinical behavior [18]. Analyses on the subgroups of GN maturing and GNBI by Cohn et al [19] and Okamatsu et al [20] supported this conclusion.

In this study, we retrospectively analyzed patients who were diagnosed with GN or GNBI in the last decade. We focused on localized stages as the typical presentations of GN and GNBI as metastatic disease is extremely rare in mature NT. Clinical features and course of GN and GNBI were compared to the group of immature localized NT. A special focus of our analysis was the outcome of patients with macroscopic tumor residuals in order to explore whether incomplete tumor resection is sufficient for the treatment of GN and GNBI.

\section{Methods}

\section{Patients and parameters}

The German neuroblastoma trials prospectively register all patients diagnosed in Germany with NT, including GN since mid of the 1990's. The German neuroblastoma trials NB97 and NB2004 were approved by the ethical committee of the University of Cologne.

For this analysis, patients were included that met the following criteria: (a) registration to the German neuroblastoma trial office with written informed consent to participate (given by the patients or their parents / guardians for patients under 18 years of age), (b) diagnosis between January 1, 2000 and December 31, 2009 with localized neuroblastic tumor, (c) age at diagnosis 21 years or younger, (d) central histological review and classification according to INPC criteria [2], (e) diagnosis of GNBI or $\mathrm{GN}$, histologically verified prior to any cytotoxic treatment. Patients with immature tumors (neuroblastoma (NB) and ganglioneuroblastoma nodular (GNBN)) that met the criteria (a) - (d) served as control. None of the patients has been included in the publication of Geoerger et al [17], while some of the patients were included in the international series published by Cohn et al [19].

Biological and clinical features and outcome were compared between mature and immature NT as well as between GN and GNBI. Tumor stage was classified according to the International Neuroblastoma Staging System (INSS) [21]. Status of MYCN oncogene and of the short arm of chromosome 1 was analyzed if a sufficient number of neuroblasts and/or ganglion cells could be analyzed in the available tumor material [22].

\section{Treatment}

For patients with GN, tumor resection without any cytotoxic treatment was recommended. Patients with NB, GNBN and GNBI were treated according to the risk stratified GPOH neuroblastoma trials NB97 and NB2004. Treatment ranged from observation to intense multimodal treatment depending on tumor stage and molecular markers. Histology was not used for treatment stratification [23-25].

For this analysis, surgical tumor removal within 3 months after diagnosis was defined as initial surgery. Operations performed after this period were defined as delayed surgery.

\section{Radiology}

Residual tumors were radiologically classified as minor or major residuals, defined by a maximum diameter of $2 \mathrm{~cm}$ in any extension in magnetic resonance imaging (MRI) as reported by local physician / radiologist. Tumor volume was calculated by the formula length * width * height / 2.

MRI series were centrally reviewed by the reference radiologist (B.K.) according to International Neuroblastoma Response Criteria (INRC) [21] and with respect to imaging quality for all patients with GN and GNBI with (suspected) tumor progression. 


\section{Statistical analysis}

Clinical features were analyzed using descriptive statistics. Differences between the groups were evaluated using the two-tailed $x^{2}$ - test, Fisher's exact test, KruskalWallis-test, and the Mann-Whitney $U$ test, whichever appropriate. Event free survival (EFS) and overall survival (OS) curves were generated using the KaplanMeier method [26] and compared by log-rank test [27]. Relapse, progression, and death of any reason were regarded as events.

\section{Results}

\section{Patient cohort}

Between January 1, 2000, and December 31, 2009, 1568 patients were registered in the German neuroblastoma trials NB97 and NB2004. 884 patients (56.4 \%) had localized tumors of which 808 patients met the inclusion criteria as described above. About one quarter showed a mature histology. In detail, 162 of 808 tumors (20.0\%) were classified as GN. The vast majority $(n=144$, $88.9 \%)$ of GN were subclassified as maturing subtype, only $18 \mathrm{GN}(11.1 \%)$ as mature subtype. Fifty-five of 808 tumors $(6.8 \%)$ were GNBI.

\section{Clinical features (Table 1)}

Patients with differentiated tumors (GN/GNBI) presented less frequently with adrenal tumors $(30.6 \%$ vs. $43.3 \%, p=0.001$ ), showed less often positive mIBGuptake $(33.6 \%$ vs. $89.6 \%, \mathrm{p}<0.001)$ and less frequently elevated urine catecholamine metabolites (homovanillic acid $38.7 \%$ vs. $61.6 \%, \mathrm{p}<0.001$, vanillylmandelic acid $26.6 \%$ vs. $64.1 \%, \mathrm{p}<0.001)$ than immature NT $(\mathrm{NB} /$ GNBN). Moreover, GN showed less often positive mIBG-uptake and elevated urine catecholamine metabolites than GNBI.

Of interest, median age at diagnosis increased with the grade of neuroblastic differentiation as impressively shown in Fig. 1. Median tumor volume at diagnosis was also larger for GN/GNBI compared to NB/GNBN (70.8 ml vs. $49.5 \mathrm{ml}, \mathrm{p}=0.001)$. Nevertheless, patients with GN/GNBI had more often stage 1 disease $(68.4 \%$ vs. $36.5 \%, \mathrm{p}<0.001$ ) than patients with immature NT. Nonetheless, $10.2 \%$ of the differentiated tumors were stage 3 and $11.5 \%$ showed intraspinal involvement.

Diagnosis of localized NT was made by routine checkups or visits to the doctor for other reasons in $50 \%$ of all cases with no significant difference between mature and immature tumors. As reported by local clinics, most frequent symptoms leading to diagnosis were pain (GN $34.2 \%$, GNBI $14.5 \%$, NB/GNBN $13.6 \%, \mathrm{p}<0.001$ ), palpable tumor mass (GN $9.3 \%$, GNBI $12.7 \%$, NB/ GNBN $18.0 \%, \mathrm{p}=0.022$ ) and reduced general condition (GN $6.2 \%$, GNBI $7.3 \%$, NB/GNBN $15.8 \%$, p = 0.003).
No amplification of MYCN was detected in 90 children with GN and 53 patients with GNBI that were analyzed. Status of $1 \mathrm{p}$ was normal in $31 \mathrm{GN}$ analyzed, while one out of 24 GNBI showed imbalance for $1 \mathrm{p}$.

\section{Surgery (Fig. 2)}

For 159 patients with GN, data on extent of initial surgery was available. Complete tumor resection was achieved within three months after diagnosis in 113 of these patients $(71.1 \%)$. Thirty-four of the 159 patients (21.4 \%) had incomplete resection and in 12 patients (7.5\%) biopsy only was performed. Hence, in 46 of 159 patients $(28.9 \%)$ residual tumor was observed for longer than three months. Twelve of these 46 patients had delayed surgery after 4 to 47 months, achieving complete resection only in four of those 12 patients. Forty-two patients with residual GN are currently under observation (median observation time: 42 months; range 1-110 months). Twenty-five of those 42 patients have major residuals $(>2 \mathrm{~cm})$, while in 14 only a minor residual was left. Information about the size of residual tumor was not available in three patients.

For 55 patients with GNBI data on extent of initial surgery was available. Tumor was completely resected within three months after diagnosis in 37 patients (67.3\%). Twelve patients (21.8 \%) had incomplete resection and six patients (10.9 \%) had biopsy only. Of those 18 patients with residual tumor, 10 underwent delayed surgery after 4 to 81 months, resulting in complete resection in five patients. Thus, in 13 patients a residual GNBI is currently under observation (median observation time: 53 months; range 6-134 months). Nine of those 13 patients have major residuals $(>2 \mathrm{~cm})$ and 4 have minor tumor residuals.

Table 2 provides more detailed information of the 22 patients with GN and GNBI who underwent delayed surgery.

\section{Chemotherapy (Table 3)}

Cytotoxic treatment was given to two patients with GN and 9 patients with GNBI. One patient with GN presented with a large stage 3 tumor and received chemotherapy because diagnosis of GN was made only from biopsy and immature components within the residuals were suspected by local physicians. The other patient with GN received chemotherapy because of an intraspinal tumor mass. No patient showed significant response to chemotherapy and residual tumor is still observed.

Nine patients with GNBI received cytotoxic treatment (stage $3 \mathrm{n}=3$; stage $2 \mathrm{a} \mathrm{n}=5$, stage $1 \mathrm{n}=1$ ). Any response to treatment was only seen in three patients. In two of these patients, tumor size slightly decreased during the first two cycles of chemotherapy, while additional chemotherapy showed no effect. The third patient did not respond to frontline chemotherapy but subsequent 
Table 1 Clinical features of the study cohort and the control group

\begin{tabular}{|c|c|c|c|c|c|c|}
\hline & $\mathrm{GN}(\mathrm{n}=162)$ & $p^{1}$ & GNBI $(n=55)$ & GN / GNBI $(n=217)$ & $\mathbf{p}^{2}$ & $\begin{array}{l}\text { NB / GNBN } \\
(\mathrm{n}=591)\end{array}$ \\
\hline \multicolumn{7}{|l|}{ Localization } \\
\hline \multirow[t]{2}{*}{ Adrenal } & $51 / 161$ & $0.613^{*}$ & $15 / 55$ & $66 / 216$ & $0.001^{*}$ & $256 / 591$ \\
\hline & $31.7 \%$ & & $27.3 \%$ & $30.6 \%$ & & $43.3 \%$ \\
\hline \multirow[t]{2}{*}{ Abdomino-pelvic } & $67 / 161$ & & $21 / 55$ & $88 / 216$ & & $211 / 591$ \\
\hline & $41.6 \%$ & & $38.2 \%$ & $40.7 \%$ & & $35.7 \%$ \\
\hline \multirow[t]{2}{*}{ Thoraco-cervical } & $43 / 161$ & & $19 / 55$ & $62 / 216$ & & $123 / 591$ \\
\hline & $26.7 \%$ & & $34.5 \%$ & $28.7 \%$ & & $20.8 \%$ \\
\hline \multicolumn{7}{|l|}{ INSS-stage } \\
\hline \multirow[t]{2}{*}{ Stage 1} & $112 / 160$ & $0.404^{* *}$ & $35 / 55$ & $147 / 215$ & $<0.001^{* *}$ & $216 / 591$ \\
\hline & $70.0 \%$ & & $63.6 \%$ & $68.4 \%$ & & $36.5 \%$ \\
\hline \multirow[t]{2}{*}{ Stage 2} & $30 / 160$ & & $16 / 55$ & $46 / 215$ & & $201 / 591$ \\
\hline & $18.8 \%$ & & $29.1 \%$ & $21.4 \%$ & & $34.0 \%$ \\
\hline \multirow[t]{2}{*}{ Stage 3} & $18 / 160$ & & $4 / 55$ & $22 / 215$ & & $174 / 591$ \\
\hline & $11.3 \%$ & & $7.3 \%$ & $10.2 \%$ & & $29.4 \%$ \\
\hline \multirow[t]{2}{*}{ Intraspinal tumor } & $16 / 162$ & 0.223 & $9 / 55$ & $25 / 217$ & 0.212 & $89 / 591$ \\
\hline & $9.9 \%$ & & $16.4 \%$ & $11.5 \%$ & & $15.1 \%$ \\
\hline Tumor Volume (median; range) & $\begin{array}{c}75 \mathrm{ml} \\
(1.6-1100)\end{array}$ & 0.228 & $\begin{array}{c}56.9 \mathrm{ml} \\
(1.5-871.9)\end{array}$ & $\begin{array}{c}70.8 \mathrm{ml} \\
(1.5-1100)\end{array}$ & 0.001 & $\begin{array}{c}49.5 \mathrm{ml} \\
(0.6-2300)\end{array}$ \\
\hline \multirow[t]{2}{*}{ Positive mIBG-uptake } & $27 / 110$ & $<0.001$ & $22 / 36$ & $49 / 146$ & $<0.001$ & $398 / 444$ \\
\hline & $24.5 \%$ & & $61.1 \%$ & $33.6 \%$ & & $89.6 \%$ \\
\hline \multirow[t]{2}{*}{ HVA elevated } & $45 / 142$ & 0.001 & $27 / 44$ & $72 / 186$ & $<0.001$ & $327 / 531$ \\
\hline & $31.7 \%$ & & $61.4 \%$ & $38.7 \%$ & & $61.6 \%$ \\
\hline Elevation of HVA above upper limit (median; range) & $\begin{array}{c}0.98 x \\
(0.22-8.1)\end{array}$ & 0.137 & $\begin{array}{c}1.46 x \\
(0.18-7.87)\end{array}$ & $\begin{array}{c}1.1 x \\
(0.18-8.1)\end{array}$ & 0.029 & $\begin{array}{c}1.5 x \\
(0.25-29)\end{array}$ \\
\hline \multirow[t]{2}{*}{ VMA elevated } & $28 / 145$ & $<0.001$ & $23 / 47$ & $51 / 192$ & $<0.001$ & $345 / 538$ \\
\hline & $19.3 \%$ & & $48.9 \%$ & $26.6 \%$ & & $64.1 \%$ \\
\hline Elevation of VMA above upper limit (median; range) & $\begin{array}{c}0.71 x \\
(0.17-16.1)\end{array}$ & 0.061 & $\begin{array}{c}1.3 x \\
(0.21-6.7)\end{array}$ & $\begin{array}{c}0.9 x \\
(0.17-16.1)\end{array}$ & $<0.001$ & $\begin{array}{c}2.1 x \\
(0.24-40.3)\end{array}$ \\
\hline \multirow[t]{2}{*}{ NSE elevated } & $50 / 124$ & 0.150 & $22 / 41$ & $72 / 165$ & $<0.001$ & $372 / 487$ \\
\hline & $40.3 \%$ & & $53.7 \%$ & $43.6 \%$ & & $76.4 \%$ \\
\hline NSE level (median; range) & $\begin{array}{l}18.3 \mathrm{ng} / \mathrm{ml} \\
(4.9-49.7)\end{array}$ & 0.706 & $\begin{array}{l}20.0 \mathrm{ng} / \mathrm{ml} \\
(9.5-49.0)\end{array}$ & $\begin{array}{l}19.0 \mathrm{ng} / \mathrm{ml} \\
(4.9-49.7)\end{array}$ & $<0.001$ & $\begin{array}{l}37.2 \mathrm{ng} / \mathrm{ml} \\
(7-2054)\end{array}$ \\
\hline \multirow[t]{2}{*}{ Diagnosis by chance } & $70 / 161$ & 0.163 & $30 / 55$ & $100 / 216$ & 0.177 & $306 / 591$ \\
\hline & $43.5 \%$ & & $54,5 \%$ & $46.3 \%$ & & $51.8 \%$ \\
\hline
\end{tabular}

$\mathrm{GN}=$ Ganglioneuroma, $\mathrm{GNBI}=$ Ganglioneuroblastoma intermixed, NB = Neuroblastoma, GNBN = Ganglioneuroblastoma nodular,

INSS = International Neuroblastoma Staging System, HVA = homovanillic acid, VMA = vanillylmandelic acid, NSE = neuron specific enolase,

${ }^{*}=$ adrenal vs. non-adrenal, ${ }^{* *}=$ stage 1 vs. stage $2 / 3, \mathrm{p}^{1}=\mathrm{p}$-value GN vs. GNBI, $\mathrm{p}^{2}=\mathrm{p}$-value GN/GNBI vs. NB/GNBN, bold $p$-values $=$ statistically significant

to incomplete tumor resection, a reduction of tumor size was observed under chemotherapy. However, no patient achieved complete or very good partial response as substantial effect of chemotherapy. In six patients who have received chemotherapy residual tumor is still under observation.

\section{Outcome}

The survival curves in Fig. 3a and 3b present the excellent prognosis of GN and GNBI. Two patients died of treatment related complications. One patient with GN died of surgery related complications after complete tumor resection in initial surgery. One patient with GNBI died of heart failure due to chemotherapy related pulmonary edema. However, no patient died of tumor progression.

Event-free survival (EFS) of patients with incomplete tumor resection was not inferior to that of patients with complete resection if tumor residuals were smaller than $2 \mathrm{~cm}$ (minor residuals; $\mathrm{p}=1.00$ ). Of interest, this applied 


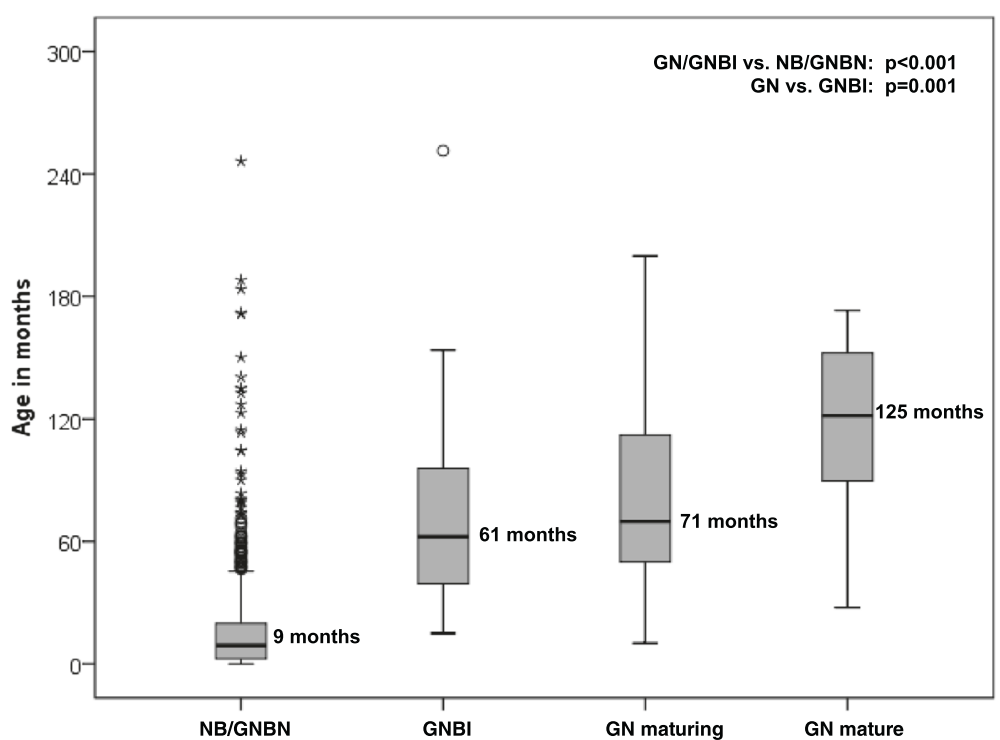

Fig. 1 Grade of differentiation in relation to median age at diagnosis. NB = neuroblastoma, GNBN = ganglioneuroblastoma nodular, $\mathrm{GNBI}=$ ganglioneuroblastoma intermixed, $\mathrm{GN}$ = ganglioneuroma
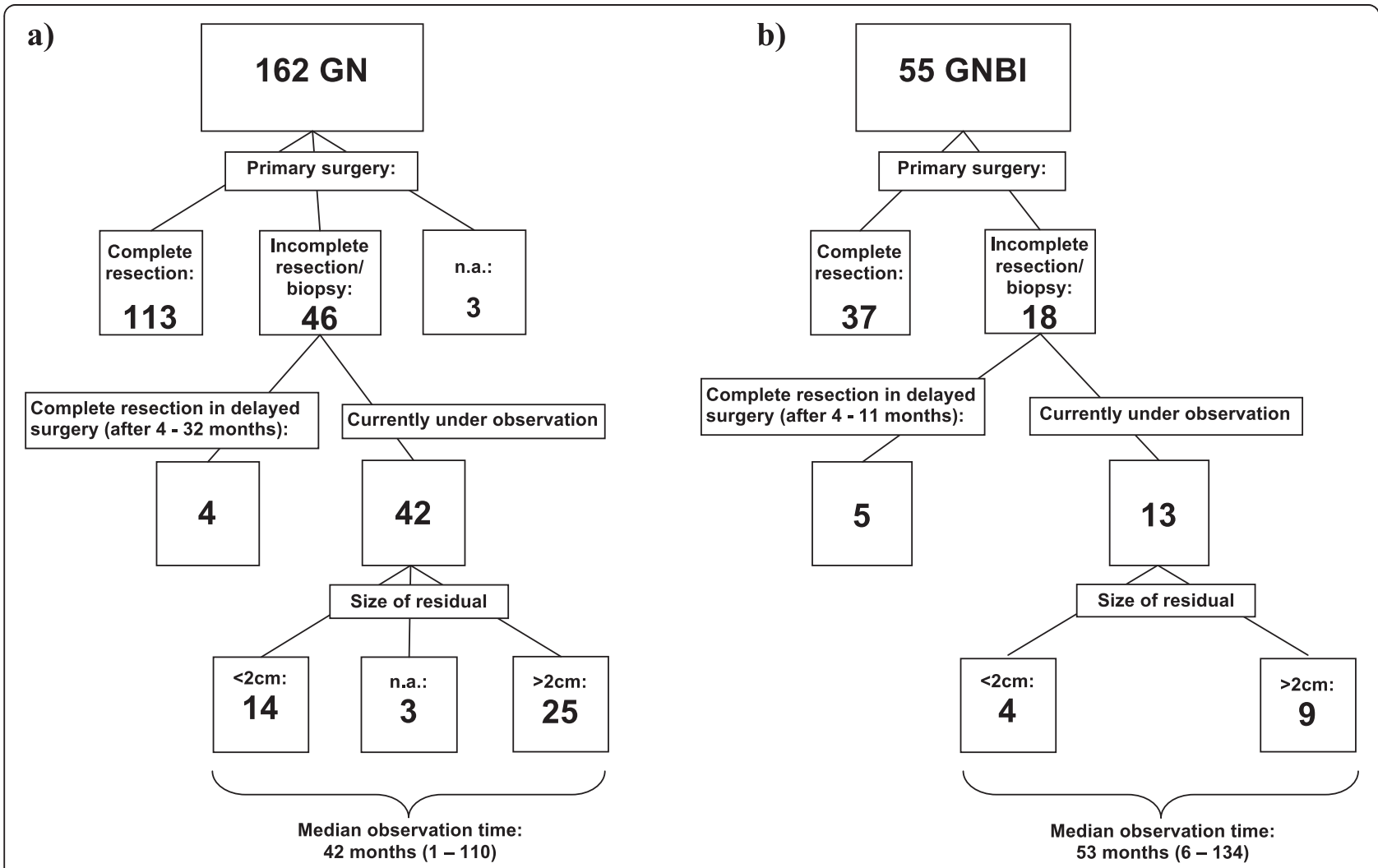

Fig. 2 Extent of initial surgeries and size of residual tumors currently under observation. GNBI = ganglioneuroblastoma intermixed,

GN = Ganglioneuroma, n.a. = data not available. a) Surgeries of patients with Ganglioneuroma, b) Surgeries of patients with Ganglioneurblastoma 
Table 2 Clinical course of patients with delayed surgery (> 3 months after diagnosis) $(n=22)$

\begin{tabular}{|c|c|c|c|c|c|c|c|c|c|}
\hline $\begin{array}{l}\text { Age at } \\
\text { diagnosis }\end{array}$ & $\begin{array}{l}\text { Tumor } \\
\text { localization }\end{array}$ & $\begin{array}{l}\text { Tumor } \\
\text { volume }\end{array}$ & $\begin{array}{l}\text { Extent of primary } \\
\text { surgery }\end{array}$ & Histology & Chemotherapy & $\begin{array}{l}\text { Time from diagnosis } \\
\text { to delayed surgery }\end{array}$ & Extent of secondary surgery & $\begin{array}{l}\text { Current } \\
\text { status }\end{array}$ & Reason for delayed surgery \\
\hline $18 \mathrm{mo}-5 \mathrm{yrs}$ & Pelvic & $179 \mathrm{ml}$ & Macroscopic residuals & GN mature & None & $32 \mathrm{mo}$ & Complete resection & $C R$ & Suspected Progression \\
\hline $11-18$ yrs & Pelvic & $680 \mathrm{ml}$ & Macroscopic residuals & GN maturing & None & $8 \mathrm{mo}$ & Macroscopic residuals & PR & Progression before delayed surgery \\
\hline $11-18$ yrs & Abdominal & n.a. & Biopsy & GN maturing & None & $4 \mathrm{mo}$ & Complete resection & $C R$ & Planned surgery \\
\hline $5-11$ yrs & Thoracic & n.a. & Macroscopic residuals & GN maturing & None & $4 \mathrm{mo}$ & Macroscopic residual & PR & Planned surgery \\
\hline $5-11$ yrs & Thoracic & $168 \mathrm{ml}$ & Macroscopic residuals & GN maturing & None & $6 \mathrm{mo}$ & Macroscopic residuals & LFU & First surgery not in Germany \\
\hline $5-11$ yrs & Abdominal & $20 \mathrm{ml}$ & Biopsy & GN maturing & None & $9 \mathrm{mo}$ & Near-complete resection & $C R$ & Suspected Progression \\
\hline $5-11$ yrs & Abdominal & $120 \mathrm{ml}$ & Macroscopic residuals & GN maturing & 2 cycles & $4 \mathrm{mo}$ & Macroscopic residual & VGPR & No response to chemotherapy \\
\hline $5-11$ yrs & Adrenal & $56 \mathrm{ml}$ & Macroscopic residuals & GN maturing & None & $5 \mathrm{mo}$ & Macroscopic residuals & VGPR & Planned surgery \\
\hline $18 \mathrm{mo}-5 \mathrm{yrs}$ & Abdominal & $90 \mathrm{ml}$ & Biopsy & GN maturing & None & $47 \mathrm{mo}$ & Macroscopic residuals & VGPR & Progression before delayed surgery \\
\hline $18 \mathrm{mo}-5 \mathrm{yrs}$ & Abdominal & $282 \mathrm{ml}$ & Biopsy & GN maturing & None & $9 \mathrm{mo}$ & Complete resection & $C R$ & Suspected Progression \\
\hline $18 \mathrm{mo}-5 \mathrm{yrs}$ & Thoracic & $16 \mathrm{ml}$ & Biopsy & GN maturing & None & $6 \mathrm{mo}$ & Macroscopic residual & VGPR & Suspected Progression \\
\hline $0-18 \mathrm{mo}$ & Thoracic & $26 \mathrm{ml}$ & Biopsy & GN maturing & None & $21 \mathrm{mo}$ & Macroscopic residuals & PR & Suspected Progression \\
\hline $18-21$ yrs & Abdominal & $72 \mathrm{ml}$ & Macroscopic residuals & GNBI & 4 cycles & $11 \mathrm{mo}$ & Biopsy & VGPR & No response to chemotherapy \\
\hline $5-11$ yrs & Thoracic & $11 \mathrm{ml}$ & Biopsy & GNBI & None & $4 \mathrm{mo}$ & Near -complete resection & $C R$ & No regression during observation \\
\hline $5-11$ yrs & Adrenal & $44 \mathrm{ml}$ & Macroscopic residuals & GNBI & 4 cycles & $5 \mathrm{mo}$ & Near-complete resection & $C R$ & No response to chemotherapy \\
\hline $5-11$ yrs & Thoracic & $42 \mathrm{ml}$ & Biopsy & GNBI & None & $11 \mathrm{mo}$ & Complete resection & $C R$ & Suspected Progression \\
\hline $18 \mathrm{mo}-5 \mathrm{yrs}$ & Adrenal & $150 \mathrm{ml}$ & Biopsy & GNBI & 4 cycles & $6 \mathrm{mo}$ & Complete resection & $C R$ & No response to chemotherapy \\
\hline $18 \mathrm{mo}-5 \mathrm{yrs}$ & Cervical & $87 \mathrm{ml}$ & Biopsy & GNBI & 4 cycles & $5 \mathrm{mo}$ & Macroscopic residual & VGPR & No response to chemotherapy \\
\hline $18 \mathrm{mo}-5 \mathrm{yrs}$ & Thoracic & n.a. & Macroscopic residuals & GNBI & 9 cycles $(+\mathrm{MT}+\mathrm{RA})$ & $17 \mathrm{mo}$ & Macroscopic residual & PR & $\begin{array}{l}\text { Clinical progression } \\
3 \text { months after diagnosis/No } \\
\text { response to chemotherapy }\end{array}$ \\
\hline $18 \mathrm{mo}-5 \mathrm{yrs}$ & Abdominal & $240 \mathrm{ml}$ & Biopsy & GNBI & 6 cycles $(+M T+R A)$ & $7 \mathrm{mo}$ & Near-complete resection & $C R$ & No response to chemotherapy \\
\hline $18 \mathrm{mo}-5 \mathrm{yrs}$ & Thoracic & $39 \mathrm{ml}$ & Biopsy & GNBI & 7 cycles & $\begin{array}{l}4 \mathrm{mo} \\
10 \mathrm{mo} \\
81 \mathrm{mo}\end{array}$ & $\begin{array}{l}\text { Macroscopic residual / } \\
\text { Macroscopic residual / Biopsy }\end{array}$ & PD & $\begin{array}{l}\text { No response to chemotherapy/ } \\
\text { Progression before surgery }\end{array}$ \\
\hline $18 \mathrm{mo}-5 \mathrm{yrs}$ & Pelvic & $98 \mathrm{ml}$ & Macroscopic residuals & GNBI & None & $22 \mathrm{mo}$ & Macroscopic residual & VGPR & Suspected Progression \\
\hline
\end{tabular}

$\mathrm{GNBI}=$ ganglioneuroblastoma intermixed, $\mathrm{GN}=$ ganglioneuroma, n.a. = data not available, yrs $=$ years, $\mathrm{mo}=$ months, $\mathrm{MT}=$ maintenance therapy, $\mathrm{RA}=$ retinoic acid,

$C R=$ complete remission, $V G P R=$ very good partial response, $P R=$ partial response, $S D=$ stable disease, $P D=$ progressive disease, $L F U=$ lost to follow-up 
Table 3 Clinical course of patients who received cytotoxic treatment (CT) $(n=11)$

\begin{tabular}{|c|c|c|c|c|c|c|c|c|c|}
\hline $\begin{array}{l}\text { Age at } \\
\text { diagnosis }\end{array}$ & $\begin{array}{l}\text { Tumor } \\
\text { localization }\end{array}$ & $\begin{array}{l}\text { Tumor } \\
\text { volume }\end{array}$ & $\begin{array}{l}\text { Tumor } \\
\text { stage }\end{array}$ & $\begin{array}{l}\text { Extent of surgery } \\
\text { prior to chemotherapy }\end{array}$ & Histology & $\mathrm{CT}$ & $\begin{array}{l}\text { Response } \\
\text { to CT }\end{array}$ & $\begin{array}{l}\text { Current } \\
\text { status }\end{array}$ & Remarks \\
\hline $11-18$ yrs & Abdominal & $768 \mathrm{ml}$ & 3 & Biopsy & GN mature & $\begin{array}{c}2 \times \mathrm{N} 5,2 \times \mathrm{N} 6 \\
5 \times \mathrm{N} 7\end{array}$ & SD & SD & $\begin{array}{l}\text { Progression } 18 \text { months } \\
\text { after end of maintenance } \\
\text { therapy (N7) }\end{array}$ \\
\hline $5-11 \mathrm{yrs}$ & Abdominal & $120 \mathrm{ml}$ & 3 & Macroscopic residuals & GN maturing & $1 \times N 5,1 \times N 6$ & SD & VGPR & $\begin{array}{l}\text { Secondary surgery due to no } \\
\text { response to chemotherapy }\end{array}$ \\
\hline $18-21$ yrs & Abdominal & $72 \mathrm{ml}$ & $2 a$ & Macroscopic residuals & GNBI & $2 \times N 5,2 \times N 6$ & SD & VGPR & $\begin{array}{l}\text { Secondary surgery due to no } \\
\text { response to chemotherapy }\end{array}$ \\
\hline $5-11 \mathrm{yrs}$ & Adrenal & $44 \mathrm{ml}$ & $2 a$ & Macroscopic residuals & GNBI & $2 \times N 5,2 \times N 6$ & SD & $C R$ & $\begin{array}{l}\text { Secondary surgery due to no } \\
\text { response to chemotherapy }\end{array}$ \\
\hline $5-11$ yrs & Pelvic & $102 \mathrm{ml}$ & $2 a$ & Macroscopic residuals & GNBI & $3 \times N 4,1 \times N 5$ & PR & LFU & LFU after 18 months \\
\hline $18 \mathrm{mo}-5 \mathrm{yrs}$ & Adrenal & $150 \mathrm{ml}$ & 3 & Biopsy & GNBI & $2 \times N 5,2 \times N 6$ & SD & $C R$ & $\begin{array}{l}\text { Secondary surgery due to no } \\
\text { response to chemotherapy }\end{array}$ \\
\hline $18 \mathrm{mo}-5 \mathrm{yrs}$ & Cervical & $87 \mathrm{ml}$ & $2 a$ & Biopsy & GNBI & $2 \times N 5,2 \times N 6$ & SD & VGPR & $\begin{array}{l}\text { Secondary surgery due to no } \\
\text { response to chemotherapy }\end{array}$ \\
\hline $18 \mathrm{mo}-5 \mathrm{yrs}$ & Thoracic & n.a. & 3 & Macroscopic residuals & GNBI & $\begin{array}{c}4 \times N 5,4 \times N 6 \\
4 \times N 7,1 \times N 8 \\
1 \times R A\end{array}$ & PR & PR & $\begin{array}{l}\text { Progression of paraplegia } 3 \\
\text { months after diagnosis }\end{array}$ \\
\hline $18 \mathrm{mo}-5 \mathrm{yrs}$ & Abdominal & $240 \mathrm{ml}$ & 3 & Biopsy & GNBI & $\begin{array}{l}3 \times \mathrm{N} 5,3 \times \mathrm{N} 6 \\
4 \times \mathrm{N} 7,9 \times \mathrm{RA}\end{array}$ & PR & $C R$ & $\begin{array}{l}\text { Secondary surgery due to } \\
\text { poor response to } \\
\text { chemotherapy }\end{array}$ \\
\hline $18 \mathrm{mo}-5 \mathrm{yrs}$ & Thoracic & $39 \mathrm{ml}$ & $2 a$ & Biopsy & GNBI & $\begin{array}{l}2 \times \mathrm{N} 5,2 \times \mathrm{N} 6 \\
2 \times \mathrm{N} 8,1 \times \mathrm{TE}\end{array}$ & SD & PD & $\begin{array}{l}\text { Progression } 71 \text { months after } \\
\text { last cycle of chemo therapy }\end{array}$ \\
\hline $0-18 \mathrm{mo}$ & Adrenal & $20 \mathrm{ml}$ & 1 & Complete resection & GNBI & $2 \times N 5,2 \times N 6$ & - & Dead & $\begin{array}{l}\text { Death of chemotherapy- } \\
\text { related pulmonary edema }\end{array}$ \\
\hline
\end{tabular}

$\mathrm{GNBI}=$ ganglioneuroblastoma intermixed, $\mathrm{GN}=$ ganglioneuroma, n.a. = data not available, yrs = years, mo = months,

$\mathrm{CR}=$ complete remission, $\mathrm{VGPR}=$ very good partial response, $\mathrm{PR}=$ partial response, $\mathrm{SD}=$ stable disease, $\mathrm{PD}=$ progressive disease, $\mathrm{LFU}=$ lost to follow-up, $\mathrm{N} 5=$ cisplatin/etopiside/vindesine, N6 = vincristine/dacarbacin/ifosfamide/doxorubicine, N7 = cyclophasphamide (oral), N8 = topotecan/cyclophasphamide/etoposide, $\mathrm{TE}=$ topotecan/etoposide

as well to the group of patients with GNBI taken by itself $(\mathrm{p}=1.00)$. However, patients with major residuals $(>2 \mathrm{~cm})$ had a worse EFS than patients with complete tumor resection or minor residuals as well for the whole group of mature NT ( $<0.001$; Fig. 4a) as for the groups of $\mathrm{GN}(\mathrm{p}=0.001$; Fig. $5 \mathrm{a})$ and GNBI seen individually ( $\mathrm{p}=0.005$; Fig. 6a). Overall survival was not influenced by extent of initial surgery (Figs. 4b, $5 b$, and $6 b)$.

Tumor progression was diagnosed in five patients (3 GN, 2 GNBI). Time to progression ranged from 3 to 80 months (median: 27 months). Four progressions were diagnosed via MRI, all of which were confirmed by central review. In three patients, progression of tumor size
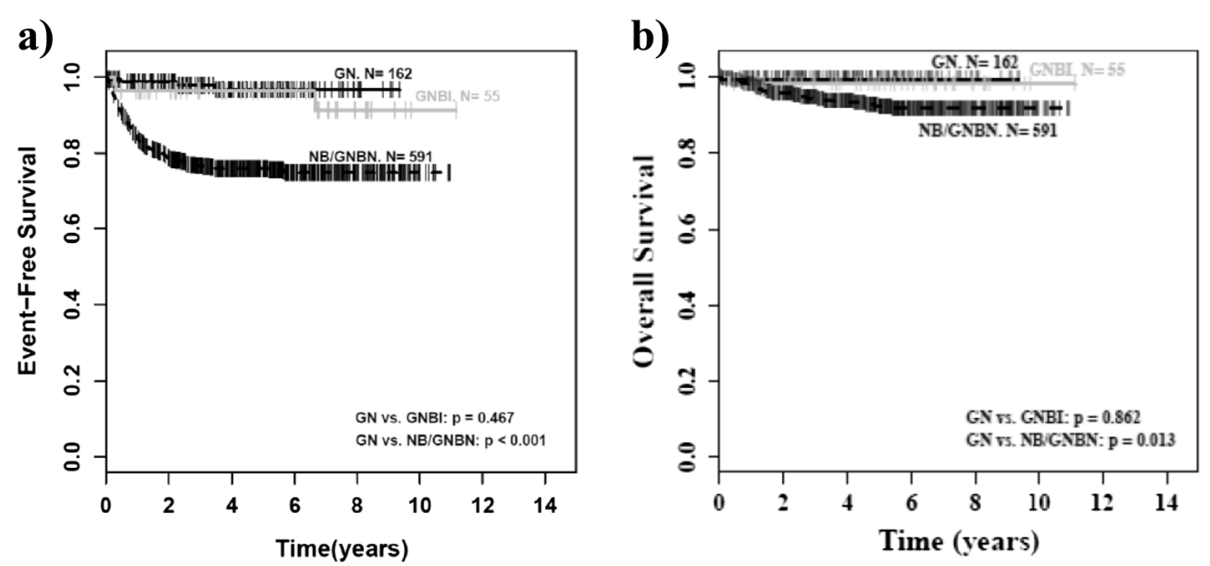

Fig. 3 Outcome by histology. NB = neuroblastoma, GNBN = ganglioneuroblastoma nodular, GNBI = ganglioneuroblastoma intermixed, GN = ganglioneuroma. a) Event-free survival (EFS) by histology, b) Overall survival (OS) by histology 

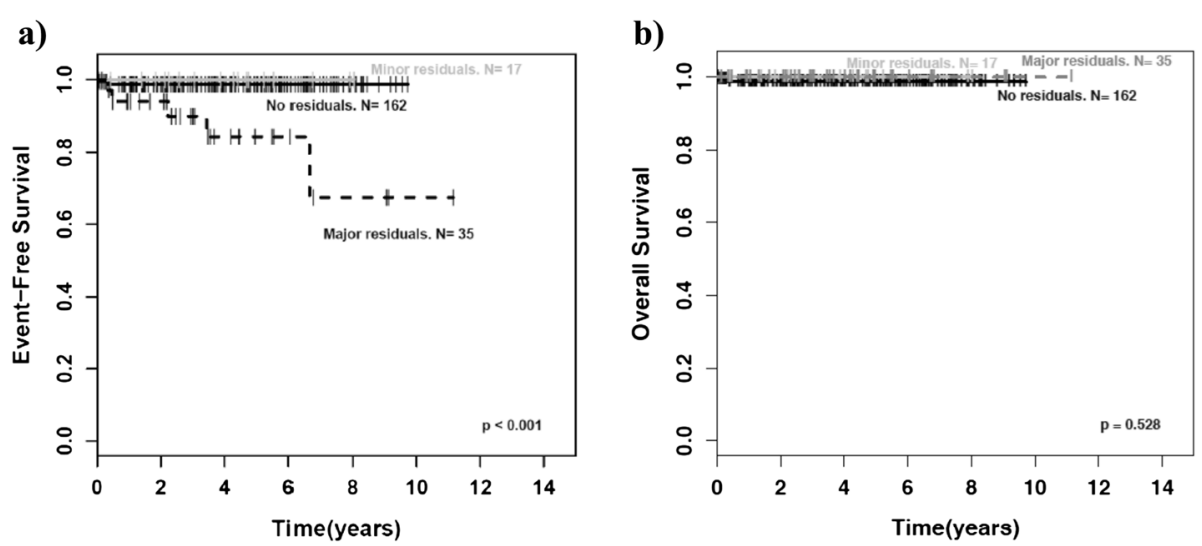

Fig. 4 Outcome of GN/GNBI by tumor residuals. GNBI = ganglioneuroblastoma intermixed, GN = ganglioneuroma. a)EFS for GN/GNBI by tumor residuals, b) OS for GN/GNBI by tumor residuals, $\mathrm{p}=$ major residuals vs. no or minor residuals

exceeded $25 \%$ as required by INRC [21]. In the fourth patient, tumor progression was diagnosed due to newly developed intraspinal involvement. The fifth progression was diagnosed in a patient with GNBI by development of progressive paraplegia after 2 cycles of chemotherapy and incomplete resection. This patient immediately underwent incomplete resection of the intraspinal tumor without performing MRI or computed tomography before surgery.

Three of five patients with progressive disease had been treated with chemotherapy prior to progression ( $1 \mathrm{GN}, 2$ GNBI). No progression was seen in the subgroup of eight out of 13 patients with residual GNBI that did not receive cytotoxic treatment.

All five patients with progression had major tumor residuals $(>2 \mathrm{~cm})$. Three of the five tumors had been initially diagnosed from biopsies. The remaining two had incomplete initial resections, both with major tumor residuals. No progression was seen after complete resection or in patients with minor tumor residuals $(<2 \mathrm{~cm})$, neither in patients with $\mathrm{GN}$ nor in patients with GNBI.

After progression was diagnosed, three patients were treated with incomplete tumor resection and one underwent biopsy. The remaining patient was further observed after first progression and even showed second progression 21 months after first progression. However, thereafter tumor has been stable. All patients with progression are still under observation with residual tumor - four with major residuals and one with a minor tumor residual. Only one of these patients received chemotherapy after progression. Further information on all patients with tumor progression is shown in Tables 2 and 3.

\section{Discussion}

This study analyzed one of the largest series of patients with GN/GNBI, demonstrating that they account for about one quarter of all localized neuroblastic tumors (NT). They show significant differences to immature NT (NB/GNBN) concerning age at diagnosis, stage, localization, tumor
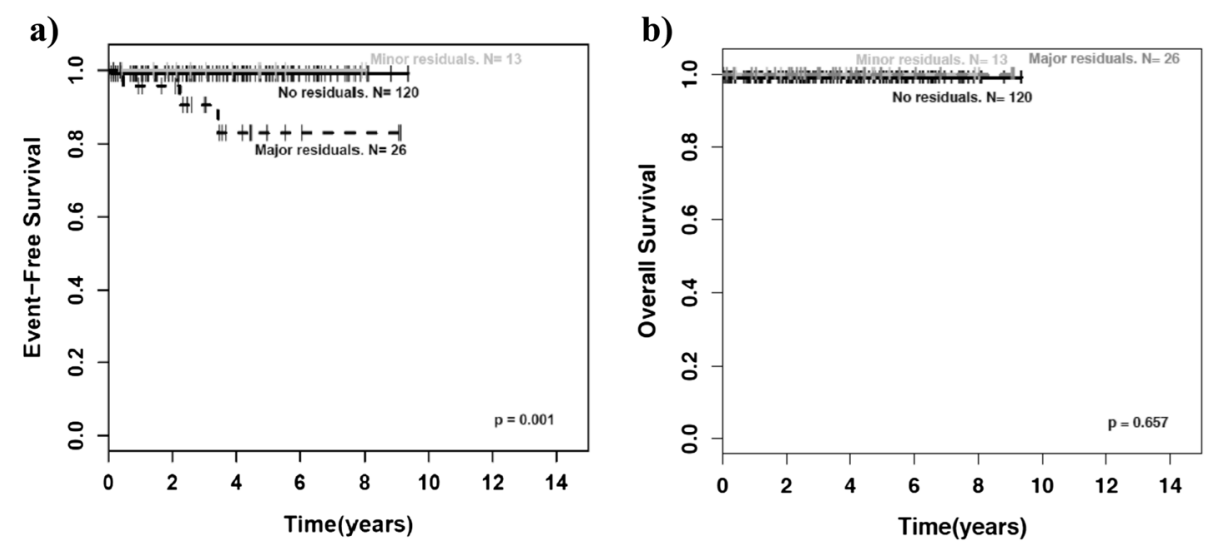

Fig. 5 Outcome of ganglioneuroma (GN) by tumor residuals. a) EFS for GN by tumor residuals, b) OS for GN by tumor residuals, $p=$ major residuals vs. no or minor residuals 

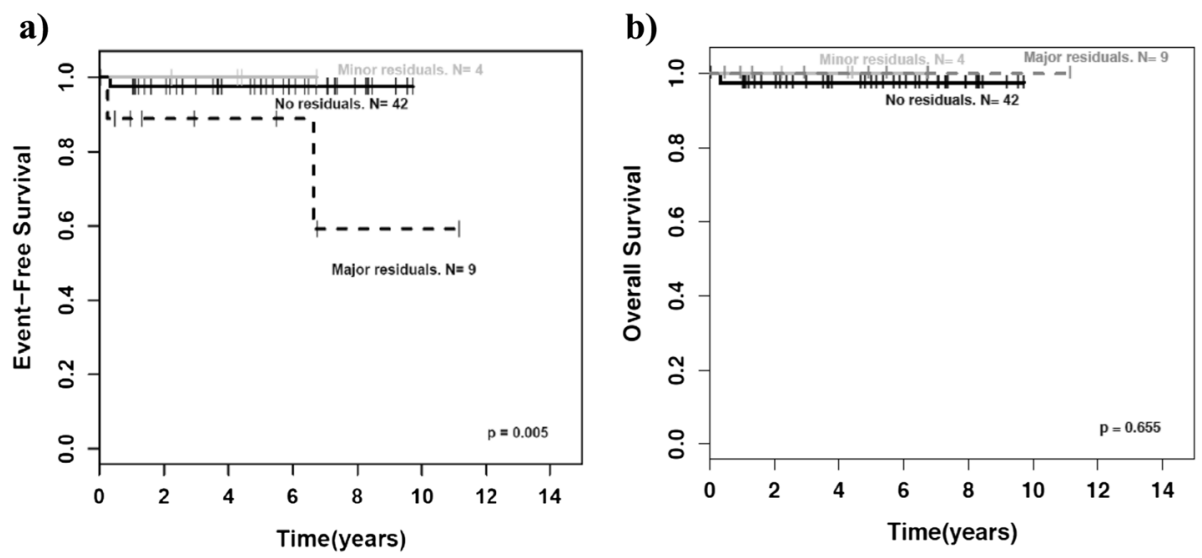

Fig. 6 Outcome of ganglioneuroblastoma intermixed (GNBI) by tumor residuals. a) EFS for GNBI by tumor residuals, b) OS for GNBI by tumor residuals, $\mathrm{p}=$ major residuals $\mathrm{vs}$. no or minor residuals

volume, mIBG-uptake, catecholamine metabolite excretion, NSE, and status of molecular markers (MYCN and 1p). Outcome was excellent and chemotherapy seems not effective while incomplete resection with minor residuals $(<2 \mathrm{~cm})$ appears to be sufficient for treatment.

In our study, the portion of GN was $20 \%$ of all localized NT which is higher than in the majority of other published studies. In their large series of GN, De Bernardi et al found $14 \%$ GN [18]. Generally, GN are likely to be under-represented in oncological trials due to the fact that benign tumors as $\mathrm{GN}$ are registered to the trial offices on a voluntary basis. We consider the higher percentage of GN observed in Germany as a reporting effect resulting from the close cooperation with the nationwide Deutsches Kinderkrebsregister (German Childhood Cancer's Registry) over the last two decades. Alignment with their data reveals that nearly all patients in Germany with NT are registered in the German neuroblastoma trials [28].

The portion of GNBI (55 of 808 localized tumors; $6.8 \%)$ appears low in our series. The ratio between GNBI $(n=55)$ and GN maturing $(n=144)$ was 1:2.6. This is much lower compared to the findings of Okamatsu et al and Cohn et al with ratios of 4.6:1 (198 GNBI, 43 GN maturing) and 3.7:1 (144 GNBI, 39 GN maturing) $[19,20]$. Nonetheless, the ratio between GNBI and all immature NT (localized or metastatic; $n=1351$ ) was 1:24.6. This is comparable to the findings of Okamatsu et al and Cohn et al where the ratio was 1:18.7 (198:3712) and 1:27 (144:3889) [19, 20]. Thus, the low portion of GNBI is artificial and results from the high completeness of included GN in our series.

Moreover, the border-line between GNBI and GN maturing - which was known as ganglioneuroblastoma borderline in the terminology proposed by Joshi et al [29] - might be set slightly different between individual pathologists. So, if central review is done by one pathologist a systematic shift to GN or GNBI might be possible.
It has been hypothesized that a good part of GN originates from neuroblastoma. This has first been suggested by Cushing in 1926 [5] and has since been suspected repeatedly for stage $4 \mathrm{~s}[11,14,30,31]$ as well as for localized neuroblastoma [24, 32]. However, whether this is true for all GN, as proposed by Shimada [2], still remains unproven. An indirect support for this hypothesis can be seen by our observation that patients with GN and GNBI are older at diagnosis than those with immature NT. As Fig. 1 shows, the grade of neuroblastic differentiation increases with the median age at diagnosis. This is in line with the theory that mature NT might just have had enough time to mature in situ before they were discovered.

Besides, the widely spread medical checkups in infants and young toddlers in Germany with the use of ultrasound could cause a higher number of asymptomatic, small localized immature neuroblastomas found in infancy before they can grow larger and potentially cause symptoms. Here, further research is needed.

Outcome was excellent with no difference between GN and GNBI. Incomplete resection was not associated with increased risk of progression if tumor residuals were smaller than $2 \mathrm{~cm}$. However, tumors that showed local progression had large tumor residuals after incomplete resection or biopsy. Therefore, it cannot be ruled out that the large tumor masses contained immature components as the sources of progression. In our study, no progression occurred after complete resection and subtotal resection with residuals $<2 \mathrm{~cm}$ in both, GN and GNBI. This supports the proposals of Duheme-Tonnelle et al [16] and Hayes et al [33] and others that resection does not have to be radical for the treatment of GN and GNBI. Instead subtotal resection without endangering vital structures seems to be sufficient. This is especially important as De Bernardi et al reported a high rate of surgical complications [18]. However, the extent of initial 
surgery should be sufficient to rule out immature areas within the tumor. Progressions only occurred in tumor residuals that were larger than $2 \mathrm{~cm}$ in diameter. However, as the overall survival of patients with progression was unimpaired after reoperation even larger tumor residuals might be acceptable to avoid risky surgery. Since progression often occurs subtle and long after initial diagnosis regular examinations of residual tumors are mandatory. Further, we found little to no benefit from cytotoxic treatment for patients with localized GNBI. Since only a very small number of patients with GNBI have received chemotherapy, it is possible that single patients may profit from cytotoxic treatment especially in case of immature regions within large tumor residuals. Our findings are in line with the results of De Bernardi et al who also demonstrated an excellent outcome for patients with GNBI without cytotoxic treatment [18]. Thus, chemotherapy seems not to be indicated for patients with localized GNBI similar as for patients with GN.

To our knowledge, this is the first analysis reporting on the influence of the size of residual tumor on EFS and the poor effect of chemotherapy in mature neuroblastic tumors.

An interesting question is why mature tumors can show progression at all. Nishihira et al suggested that a minor increase of tumor volume may also be caused by proliferation of Schwannian stroma rather than that of tumor cells [34]. This may explain limited variations in tumor volume not reaching $25 \%$ as required for diagnosis of tumor progression according to INRC [21].

\section{Conclusions}

In conclusion, clinical features and behavior are very similar for GN and GNBI and outcome is excellent for both. Cytotoxic treatment has no substantial effect on both tumor entities. Surgery alone is sufficient for the treatment of GN and GNBI and does not need to be radical if only minor residuals are left (e.g. $<2 \mathrm{~cm}$ ). For patients diagnosed with GN or GNBI, subtotal, non-mutilating resection and regular long-term follow up are warranted.

\section{Additional file}

Additional file 1: Dataset (PDF $291 \mathrm{~kb})$

\footnotetext{
Abbreviations

1p, Short arm of chromosome 1; EFS, Event-free survival; GN, Ganglioneuroma; GNBI, Ganglioneuroblastoma intermixed; GNBN, Ganglioneuroblastoma nodular; $\mathrm{GPOH}$, German Association for Pediatric Oncology and Hematology (Gesellschaft für pädiatrische Onkologie und Hämatologie); INPC, International Neuroblastoma Pathology Classification; INRC, International Neuroblastoma Response Criteria; INSS, International Neuroblastoma Staging System; mIBG, Metaiodobenzylguanidine; MRI, Magnetic resonance imaging; MYCN, Oncogene MYCN; NB, Neuroblastoma; NSE, Neuron specific enolase; NT, Neuroblastic tumors; OS, Overall survival
}

\section{Acknowledgements}

The German neuroblastoma trials were supported by the Deutsche Krebshilfe (NB97: \#102546; NB2004: \#107712). The authors thank the staff at the trial office in Cologne and all documenting colleagues in the participating hospitals.

\section{Funding}

The German neuroblastoma trials were supported by the Deutsche Krebshilfe (NB97: \#102546; NB2004: \#107712).

\section{Availability of data and materials}

See additional supporting file Additional file 1: "Dataset".

\section{Authors' contributions}

$\mathrm{BD}$ performed study design, statistical analysis, revision of collected data, coordination of histological and pathological review, and drafting and finalizing the manuscript. TS contributed to data acquisition, study design, and drafting the manuscript. BK performed central radiological review according to INRC. IL and CV performed central histological review and classification according to INPC criteria. PK, DVS, TK, IM, and LS substantially contributed to acquisition of clinical data. FB contributed to data acquisition and study design and was involved in drafting the manuscript. $\mathrm{BH}$ substantially contributed to study design, data base, statistical analysis and drafting and finalizing the manuscript. All authors have read, revised, and approved the final manuscript.

\section{Competing interests}

The authors declare that they have no competing interests.

\section{Consent for publication}

Not applicable.

\section{Ethics approval and consent to participate}

The German neuroblastoma trials NB97 and NB2004 were approved by the ethical committee of the University of Cologne. All patients included in our analysis were registered to the German neuroblastoma trial office with written informed consent to participate in the study (given by the patients or their parents / guardians for patients under 18 years of age).

\section{Author details \\ 'Department of Pediatric Hematology and Oncology, Children's Hospital, University of Cologne, Cologne, Germany. 'Department of Radiology, University of Cologne, Cologne, Germany. ${ }^{3}$ Department of Pathology, University of Kiel, Kiel, Germany. ${ }^{4}$ German Childhood Cancer Registry, University of Mainz, Mainz, Germany. ${ }^{5}$ Department of Pediatric Surgery, Dr von Hauner Children's Hospital, Ludwig-Maximilians-University Munich, Munich, Germany. ${ }^{6}$ Clinic for Pediatric Hematology and Oncology, Johann Wolfgang Goethe-University, Frankfurt, Germany. 'Department of Pediatric Hematology and Oncology, University Medical Center Hamburg-Eppendorf, Hamburg-Eppendorf, Germany. ${ }^{8}$ Clinic for Pediatrics, Helios Klinikum Berlin-Buch, Berlin-Buch, Germany.}

Received: 22 July 2015 Accepted: 27 June 2016

Published online: 27 July 2016

\section{References}

1. Kaatsch P. Epidemiology of childhood cancer. Cancer Treat Rev. 2010. doi: 10.1016/j.ctrv.2010.02.003

2. Shimada H, Ambros IM, Dehner LP, Hata J, Joshi W, Roald B. Terminology and morphologic criteria of neuroblastic tumors: recommendations by the International Neuroblastoma Pathology Committee. Cancer. 1999;86(2):349-63. doi:10.1002/(SICI)1097-0142(19990715)86:2<349::AID-CNCR20>3.0.CO;2-Y.

3. Mora J, Gerald WL. Origin of neuroblastic tumors: clues for future therapeutics. Expert Rev Mol Diagn. 2004;4(3):293-302. doi:10.1586/14737159.4.3.293.

4. McFarland J, Sappington SW. A Ganglioneuroma in the neck of a child. Am J Pathol. 1935;11(3):429-48. 1.

5. Cushing $\mathrm{H}$, Wolbach SB. The transformation of a malignant paravertebral sympathicoblastoma into a Benign Ganglioneuroma. Am J Pathol. 1927:3(3):203-16. 7.

6. Sophian L. Mediastinal Ganglioneuroma. Ann Surg. 1935;101(3):827-33. 
7. Moschovi M, Arvanitis D, Hadjigeorgi C, Mikraki V, Tzortzatou-Stathopoulou F. Late malignant transformation of dormant ganglioneuroma? Med Pediatr Oncol. 1997;28(5):377-81. 10.1002/(SICI)1096-911X(199705)28:5<377::AIDMPO11>3.0.CO;2-8.

8. Kulkarni AV, Bilbao JM, Cusimano MD, Muller PJ. Malignant transformation of ganglioneuroma into spinal neuroblastoma in an adult. Case report. J Neurosurg. 1998;88(2):324-7. doi:10.3171/jns.1998.88.2.0324.

9. Schulman H, Laufer L, Barki Y, Philip M, Mares AJ, Maor E, et al. Ganglioneuroma: an 'incidentaloma' of childhood. Eur Radiol. 1998;8(4):582-4

10. Cronin EM, Coffey JC, Herlihy D, Romics L, Aftab F, Keohane C, et al. Massive retroperitoneal ganglioneuroma presenting with small bowel obstruction 18 years following initial diagnosis. Ir J Med Sci. 2005;174(2):63-6.

11. Przkora R, Perez-Canto A, Ertel W, Heyde CE. Ganglioneuroma : primary tumor or maturation of a suspected neuroblastoma? Eur Spine J. 2006;15(3):363-5. doi:10.1007/s00586-005-0964-9.

12. Ponce-Camacho MA, Diaz de Leon-Medina R, Miranda-Maldonado I, GarzaGuajardo R, Hernandez-Salazar J, Barboza-Quintana O. A 5-year-old girl with a congenital ganglioneuroma diagnosed by fine needle aspiration biopsy: a case report. Cytojournal. 2008;5:5. doi:10.1186/1742-6413-5-5.

13. Arce Gil J, Arce Terroba Y, Angerri Feu O, Caffaratti Sfulcini J, Garat Barredo $\mathrm{JM}$, Villavicencio MH. Retroperitoneal ganglioneuroma in the infancy. Actas Urol Esp. 2008;32(5):567-70.

14. Rozmus J, Langer M, Murphy JJ, Dix D. Multiple persistent ganglioneuromas likely arising from the spontaneous maturation of metastatic neuroblastoma. J Pediatr Hematol Oncol. 2012;34(2):151-3. doi:10.1097/MPH Ob013e318221ca82.

15. Geraci AP, de Csepel J, Shlasko E, Wallace SA. Ganglioneuroblastoma and ganglioneuroma in association with neurofibromatosis type I: report of three cases. J Child Neurol. 1998;13(7):356-8.

16. Duhem-Tonnelle V, Vinchon M, Defachelles AS, Cotten A, Dhellemmes P. Mature neuroblastic tumors with spinal cord compression: report of five pediatric cases. Childs Nerv Syst. 2006;22(5):500-5. doi:10.1007/s00381-005-0009-z.

17. Geoerger B, Hero B, Harms D, Grebe J, Scheidhauer K, Berthold F. Metabolic activity and clinical features of primary ganglioneuromas. Cancer. 2001; 91(10):1905-13. doi:10.1002/1097-0142(20010515)91:10<1905::AIDCNCR1213>3.0.CO:2-4

18. De Bernardi B, Gambini C, Haupt R, Granata C, Rizzo A, Conte M, et al. Retrospective study of childhood ganglioneuroma. J Clin Oncol. 2008;26(10):1710-6. doi:10.1200/JCO.2006.08.8799.

19. Cohn SL, Pearson AD, London WB, Monclair T, Ambros PF, Brodeur GM, et al. The International Neuroblastoma Risk Group (INRG) classification system: an INRG Task Force report. J Clin Oncol. 2009;27(2):289-97. doi:10.1200/JCO.2008.16.6785.

20. Okamatsu C, London WB, Naranjo A, Hogarty MD, Gastier-Foster JM, Look AT, et al. Clinicopathological characteristics of ganglioneuroma and ganglioneuroblastoma: a report from the CCG and COG. Pediatr Blood Cancer. 2009;53(4):563-9. doi:10.1002/pbc.22106.

21. Brodeur GM, Pritchard J, Berthold F, Carlsen NL, Castel V, Castelberry RP, et al. Revisions of the international criteria for neuroblastoma diagnosis, staging, and response to treatment. J Clin Oncol. 1993;11(8):1466-77.

22. Spitz R, Hero B, Ernestus K, Berthold F. FISH analyses for alterations in chromosomes 1, 2, 3, and 11 define high-risk groups in neuroblastoma. Med Pediatr Oncol. 2003:41(1):30-5. doi:10.1002/mpo.10313.

23. Berthold F, Boos J, Burdach S, Erttmann R, Henze G, Hermann J, et al. Myeloablative megatherapy with autologous stem-cell rescue versus oral maintenance chemotherapy as consolidation treatment in patients with high-risk neuroblastoma: a randomised controlled trial. Lancet Oncol. 2005;6(9):649-58. doi:10.1016/S1470-2045(05)70291-6.

24. Hero B, Simon T, Spitz R, Ernestus K, Gnekow AK, Scheel-Walter HG, et al. Localized infant neuroblastomas often show spontaneous regression: results of the prospective trials NB95-S and NB97. J Clin Oncol. 2008;26(9):1504-10. doi:10.1200/JCO.2007.12.3349

25. Simon T, Spitz R, Faldum A, Hero B, Berthold F. New definition of low-risk neuroblastoma using stage, age, and $1 p$ and MYCN status. J Pediatr Hematol Oncol. 2004;26(12):791-6.

26. Kaplan EL, Meier P. Nonparametric Estimation from Incomplete Observations. J Am Stat Assoc. 1958;53(282):457-81.

27. Coldman AJ, Elwood JM. Examining survival data. Can Med Assoc J. 1979; 121(8):1065-8. 71.

28. Kaatsch P, Spix J. German Childhood Cancer Registry - Report 2013/2014 (1980-2013). Institute of Medical Biostatistics, Epidemiology and Informatics
(IMBEI) at the University Medical Center of the Johannes Gutenberg University Mainz; 2014. http://www.kinderkrebsregister.de/typo3temp/ secure_downloads/26650/0/22cb72c5a76a0e46c1fe00358768d9ab8ecd3c1e/ jb2014_s.pdf.

29. Joshi W, Cantor AB, Altshuler G, Larkin EW, Neill JS, Shuster JJ, et al. Recommendations for modification of terminology of neuroblastic tumors and prognostic significance of Shimada classification. A clinicopathologic study of 213 cases from the Pediatric Oncology Group. Cancer. 1992;69(8): 2183-96.

30. Haas D, Ablin AR, Miller C, Zoger S, Matthay KK. Complete pathologic maturation and regression of stage IVS neuroblastoma without treatment. Cancer. 1988;62(4):818-25.

31. Garvin Jr JH, Lack EE, Berenberg W, Frantz CN. Ganglioneuroma presenting with differentiated skeletal metastases. Report of a case. Cancer. 1984;54(2): 357-60.

32. Eyre-Brook AL, Hewer TF. Spontaneous disappearance of neuroblastoma with maturation to ganglioneuroma. J Bone Joint Surg Br. 1962;44-B(4):886-90.

33. Hayes FA, Green AA, Rao BN. Clinical manifestations of ganglioneuroma. Cancer. 1989;63(6):1211-4

34. Nishihira H, Toyoda Y, Tanaka Y, ljiri R, Aida N, Takeuchi M, et al. Natural course of neuroblastoma detected by mass screening: $s$ 5-year prospective study at a single institution. J Clin Oncol. 2000;18(16):3012-7.

\section{Submit your next manuscript to BioMed Central and we will help you at every step:}

- We accept pre-submission inquiries

- Our selector tool helps you to find the most relevant journal

- We provide round the clock customer support

- Convenient online submission

- Thorough peer review

- Inclusion in PubMed and all major indexing services

- Maximum visibility for your research

Submit your manuscript at www.biomedcentral.com/submit
Biomed Central 